\title{
Potential for tyndalized Lactobacillus acidophilus as an effective component in moisturizing skin and anti-wrinkle products
}

\author{
A-RANG IM ${ }^{1}$, HUI SEONG KIM ${ }^{2}$, JIN WON HYUN ${ }^{3}$ and SUNGWOOK CHAE ${ }^{1}$ \\ ${ }^{1}$ KM Convergence Research Division, Korea Institute of Oriental Medicine, Daejeon 305-811; \\ ${ }^{2}$ Division of AIDS, Center for Immunology and Pathology, Korea National Institute of Health, Cheongju, Chungbuk 363-951; \\ ${ }^{3}$ School of Medicine and Institute for Nuclear Science and Technology, \\ Jeju National University, Jeju-si, Jeju-do 690-756, Republic of Korea
}

Received March 26, 2015; Accepted April 25, 2016

DOI: $10.3892 /$ etm.2016.3406

\begin{abstract}
It is widely accepted that ultraviolet (UV) irradiation induces skin damage. In the present study, a UVB-induced hairless mouse model of skin photoaging was developed to determine whether tyndalized Lactobacillus acidophilus was able to significantly enhance the repair of photodamaged skin. To evaluate the effects of tyndalized L. acidophilus on UVB-induced skin-wrinkle formation in vivo, HR-1 hairless male mice were exposed to UVB radiation and orally administered tyndalized L. acidophilus. Compared with the control group, the UVB irradiation mice displayed a significant increase in transepidermal water loss and a reduction in skin hydration. In mice with UVB-induced photodamage, the effacement of the fine wrinkles by tyndalized L. acidophilus was correlated with dermal collagen synthesis, accompanied by histological changes. Furthermore, western blotting was performed to investigate the protein expression levels of matrix metalloproteinases (MMPs) and mitogen-activated protein kinase. Notably, orally administered tyndalized L. acidophilus reduced the expression levels of MMP-1 and MMP-9. Based upon the aforementioned results, it was determined that tyndalized L. acidophilus effectively inhibited the wrinkle formation induced by UVB irradiation, and that this
\end{abstract}

Correspondence to: Dr Sungwook Chae, KM Convergence Research Division, Korea Institute of Oriental Medicine, 1672 Yuseongdae-Ro, Yuseong-Gu, Daejeon 305-811, Republic of Korea

E-mail:kendall@kiom.re.kr

Abbreviations: ECM, extracellular matrix; ERK, extracellular signal-regulated protein kinases; H\&E, hematoxylin and eosin; MAPK, mitogen-activated protein kinase; MEK, mitogen-activated protein kinase/extracellular signal-regulated protein kinase; MMP, matrix metalloproteinase; JNK Jun-amino-terminal kinase; TEWL, transepidermal water loss; UV ultraviolet

Key words: anti-wrinkle, tyndalized Lactobacillus acidophilus, transepidermal water loss, matrix metalloproteinases, mitogen-activated protein kinase may be attributed to the downregulation of MMPs. Therefore, tyndalized L. acidophilus may be considered a potential agent for preventing skin photoaging and wrinkle formation.

\section{Introduction}

Skin is in continuous contact with the external environment, and protects the human body from potentially hazardous environmental threats, including physical, chemical and biochemical factors (1). Furthermore, skin prevents the entry of bacteria, fungi and viruses into the body, and provides a protective barrier that prevents moisture loss (2). Skin aging comprises several intrinsic processes, which are predominately genetically determined, and extrinsic aging that is typically associated with sun exposure; however, other factors may be involved in extrinsic skin aging, including ultraviolet (UV) irradiation, excessive alcohol consumption and environmental pollution $(1,2)$.

Chronic exposure of human skin to the sun is characterized by epidermal hyperplasia and changes in the biomechanical properties of the dermis (3). These lead to wrinkle formation that may be observed histologically, and the development of deep wrinkles, nodules, irregular hyperpigmentation, telangiectasia and skin that has a leathery, rough texture, all of which are clinically evident (3).

Repeated exposure to UV radiation ultimately causes premature skin aging, or photoaging, which is characterized by the formation of fine and coarse wrinkles, an increase in the thickness of the skin, dryness, laxity and pigmentation (4). Exposure of the skin to UV light initiates the generation of active oxygen species in the skin (5), and exposure of the skin to UVB radiation is associated with elevated risks of erythema, edema, hyperplasia, sunburn-cell formation, photoaging, immune system suppression and skin cancer (6).

The role of probiotics in the regulation of intestinal health has been widely investigated for over 100 years. Probiotics are used with increasing frequency to treat medical conditions such as allergic diseases and atopic dermatitis, and to prevent dental caries and respiratory infections (7). Human clinical trials have demonstrated that probiotic supplementation may relieve atopic dermatitis and dry skin (8). Several studies involving Lactobacillus acidophilus have demonstrated 
that probiotics are effective against atopic dermatitis $(9,10)$. However, the anti-wrinkle effects of tyndalized L.acidophilus have not been investigated. Thus, the present study examined the effects of tyndalized L.acidophilus on hairless mice that had developed skin damage following skin exposure to UVB radiation.

\section{Materials and methods}

Materials. Tyndalized L. acidophilus, or ID-ACT3302, was obtained from Ildong Pharmaceutical Co., Ltd. (Seoul, South Korea). A total of 21 6-week-old HR-1 hairless male mice were purchased from Japan SLC, Inc. (Shizuoka, Japan). UVB irradiation was administered using a UVM-225D Mineralight UV Display Lamp (UVP, Inc., Upland, CA, USA). Rabbit anti-phospho-stress-activated protein kinase/Jun-amino-terminal kinase (SAPK/JNK; cat. no. 9251), anti-SAPK/JNK (cat. no. 9252), anti-phospho-p44/42 mitogen-activated protein kinase (MAPK) extracellular signal-regulated protein kinases 1 and 2(ERK1/2; cat. no. 9101), anti-p44/42 MAPK (ERK1/2; cat. no. 9102), anti-phospho-p38 MAPK (cat. no. 9211) and anti-p38 MAPK (cat. no. 9212) polyclonal antibodies were purchased from Cell Signaling Technology (Danvers, MA, USA). Goat anti-matrix metalloproteinase (MMP)-1 polyclonal antibody (cat. no. sc12348), mouse anti-MMP-9 monoclonal antibody (cat. no. sc-21733), goat anti- $\beta$-actin polyclonal antibody (cat. no. sc-1616) and horseradish peroxidase (HRP)-conjugated anti-rabbit (cat. no. sc-2030) and anti-goat (cat. no. sc-2020) secondary antibodies were purchased from Santa Cruz Biotechnology, Inc. (Dallas, TX, USA).

Experimental animals and oral administration. After purchase, the HR-1 hairless male mice were stabilized for 1 week prior to the commencement of the study. The animals were housed in a climate-controlled facility at a temperature of $24^{\circ} \mathrm{C}$, at $50 \%$ humidity with a 12 -h dark:light cycle and ad libitum access to food and water. All experimental protocols were approved by the Korea Institute of Oriental Medicine Institutional Animal Care and Use Committee (Daejeon, South Korea). The mice were divided into 3 groups as follows: Control $(n=5)$, UVB-treated vehicle $(n=5)$, and UVB-treated tyndalized L. acidophilus $(\mathrm{n}=5)$ groups. Mice from the UVB-treated tyndalized L. acidophilus group were orally administered $0.1 \mathrm{ml}$ water containing $100 \mathrm{mg}$ of tyndalized L. acidophilus/kg body weight/day. The control group did not receive irradiation with UVB, or any other form of treatment. The vehicle group mice were orally administered $0.1 \mathrm{ml}$ water and underwent irradiation with UVB.

UVB irradiation. UVB irradiation was administered using a UVM-225D Mineralight UV Display Lamp (UVP) that emitted at a wavelength of $302 \mathrm{~nm}$. The strength of the UV radiation was measured using a HD2102-2 UV meter (Delta OHM Srl, Padova, Italy). UVB radiation was applied to the backs of the mice 3 times/week for 12 weeks. The amount of irradiation was progressively increased from $60 \mathrm{~mJ} / \mathrm{cm}^{2} /$ exposure during week 1 ( 1 minimal erythematous dose $=60 \mathrm{~mJ} / \mathrm{cm}^{2}$ ) to $90 \mathrm{~mJ} / \mathrm{cm}^{2} /$ exposure during week 7 .
A

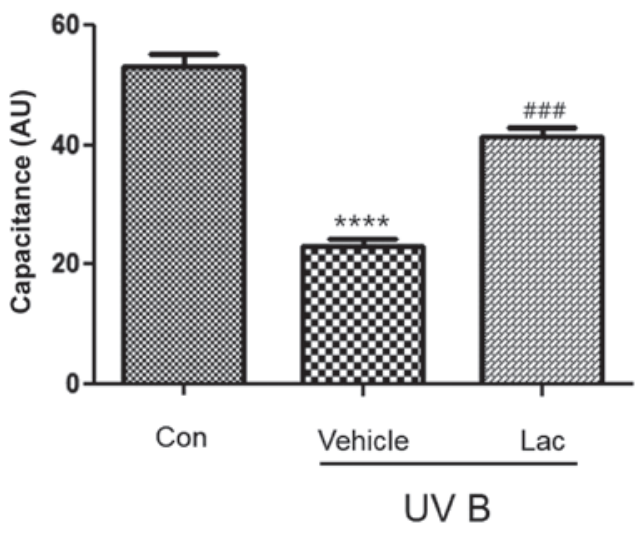

B

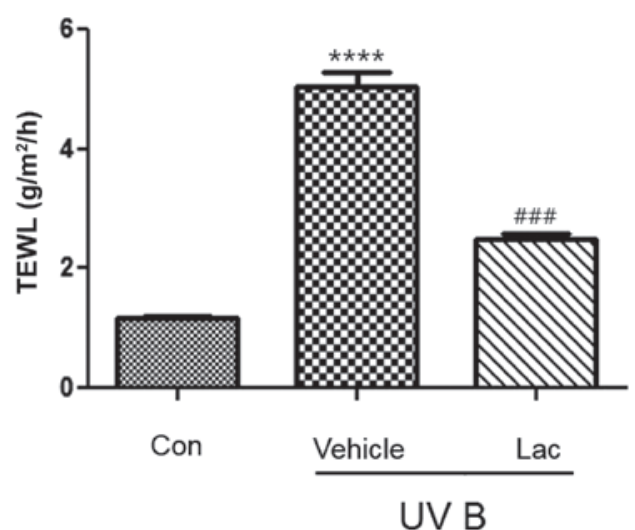

Figure 1. Effect of tyndalized Lac on skin hydration and TEWL. (A) Skin hydration was significantly reduced following UVB irradiation, as compared with the control group. Tyndalized Lac significantly increased skin hydration, as compared with the vehicle-treated group. (B) TEWL was significantly increased following UVB irradiation, as compared with the control group. Tyndalized Lac significantly decreased TEWL, as compared with the vehicle-treated group. Data are presented as the mean \pm standard error. ${ }^{* * * * *} \mathrm{P}<0.0001$ vs. the control group. ${ }^{\# \#} \mathrm{P}<0.001$ vs. the vehicle group. Lac, Lactobacillus acidophilus; TEWL, transepidermal water loss; UV, ultraviolet.

Skin hydration and transepidermal water loss (TEWL). A corneometer (Courage + Khazaka electronic GmbH, Cologne, Germany) was used to determine the hydration levels of the skin, and a Tewameter (Courage + Khazaka electronic GmbH) was used to measure TEWL, which is an indicator of the barrier function of the skin's epidermis.

Histological investigation. The dorsal skin was removed from each hairless mouse and fixed in 10\% neutral-buffered formalin. Using a conventional method, the fixed tissue samples were washed with distilled water, dehydrated using an ethanol gradient, cleared with xylene and embedded in paraffin wax, after which $5 \mu \mathrm{m}$ sections were cut using a microtome. The tissue sections were stained with hematoxylin and eosin (H\&E) and Masson's trichrome stain for collagen fiber analysis. The thickness of the epidermis was measured under light microscopy using an eyepiece micrometer (Olympus Corporation, Tokyo, Japan).

Western blotting. Protein was extracted from the skin tissue samples using radioimmunoprecipitation assay lysis buffer 
A

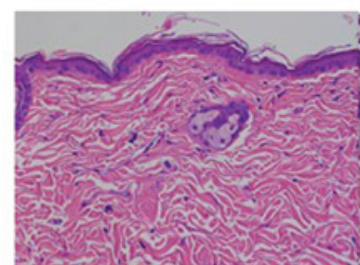

Control

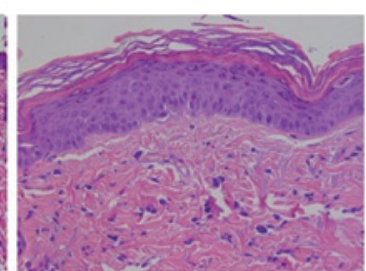

Vehicle

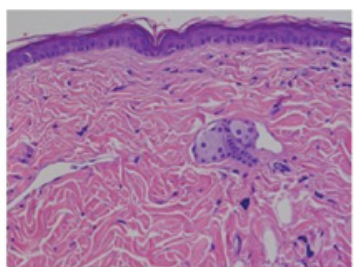

Lac

UVB

B

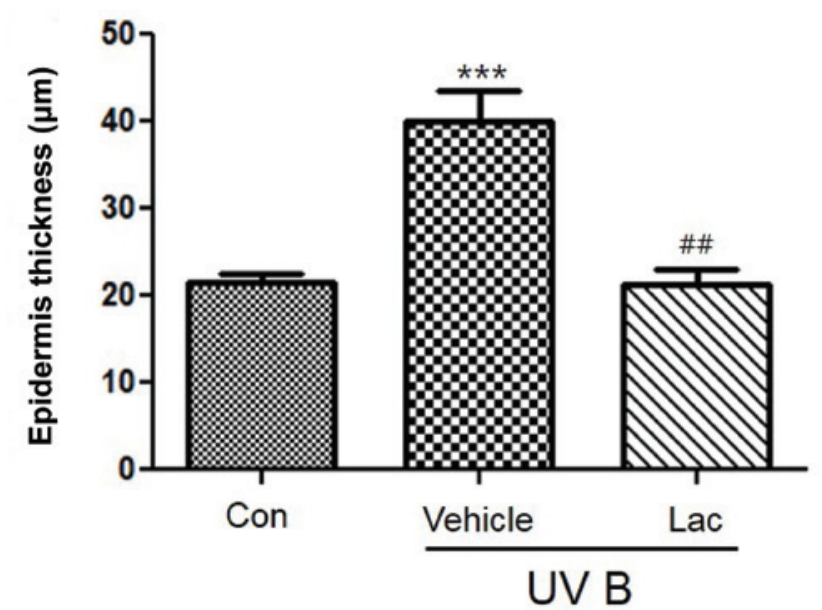

C

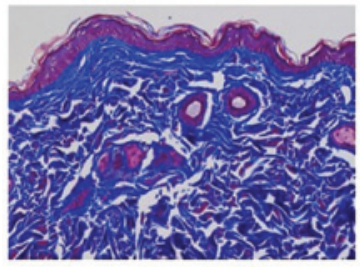

Control

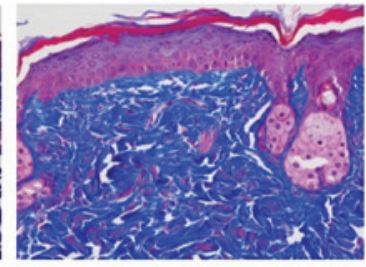

Vehicle

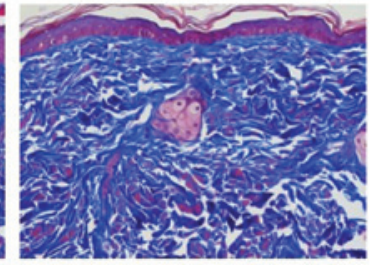

Lac

UVB

Figure 2. Tyndalized Lactobacillus acidophilus treatment reduced UVB-induced skin thickening in hairless mice. (A) Tyndalized L. acidophilus suppressed the UVB-induced increase in epidermal thickness. Hematoxylin and eosin staining of UVB-irradiated hairless mice skin. Magnification, x200. (B) Epidermal thickness of the dorsal skin. Data are presented as the mean \pm standard error. ${ }^{* * *} \mathrm{P}<0.001$ vs. the control group. ${ }^{\# \#} \mathrm{P}<0.01$ vs. the vehicle group. (C) Protective effect of tyndalized L.acidophilus against changes in the number of collagen fibers. Histological staining of hairless mouse skin stained with Masson's trichrome stain. Collagen fibers are stained blue. Magnification, x200. Lac, tyndalized L acidophilus; UVB, ultraviolet B.

(Thermo Fisher Scientific, Inc., Rockford, IL, USA), following tissue homogenization (Precellys ${ }^{\circledR} 24$; Bertin Technologies, Montigny-le-Bretonneux, France). Total protein concentration was determined using the DC Protein Assay (Bio-Rad Laboratories, Inc., Hercules, CA, USA). Protein lysates (20 $\mu \mathrm{g})$ from each sample were electrophoresed on a $10 \%$ sodium dodecyl sulfate-polyacrylamide gel and then transferred to polyvinylidene fluoride membranes. The membranes were blocked with a $5 \%$ skimmed milk solution for $1 \mathrm{~h}$ at room temperature. Following blocking, the blots were incubated overnight at $4{ }^{\circ} \mathrm{C}$ with a the primary antibodies at a dilution of 1:1,000. The blots were washed 3 times for $10 \mathrm{~min}$ each in Tris-buffered saline (Bio-Rad Laboratories, Inc.). The membranes were then incubated for $2 \mathrm{~h}$ with HRP-conjugated anti-rabbit and anti-goat secondary antibodies at a dilution of 1:5,000. The proteins were detected using an enhanced chemiluminescence solution. Images were captured by
ImageQuant LAS 4000 digital imaging system (GE Healthcare Bio-Sciences, Pittsburgh, PA, USA) and were analyzed using Multi Gauge software, version 3.0 (Fujifilm, Tokyo, Japan).

Statistical analysis. All measurements were undertaken in triplicate, and all values are presented as the mean \pm standard error. An analysis of variance and Tukey's test was used to determine differences in the results among the study groups. Statistical analyses were performed using GraphPad Prism 5.0 software (GraphPad Software, Inc., La Jolla, CA, USA). P<0.05 was considered to indicate a statistically significant difference.

\section{Results}

Evaluation of TEWL and skin hydration. The hydration of the stratum corneum was significantly reduced in the UVB-treated vehicle group, as compared with the control group $(\mathrm{P}<0.0001$; 
A

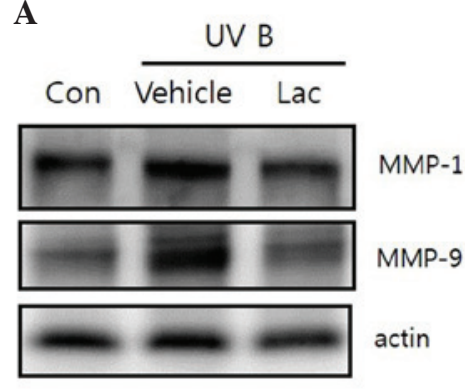

B

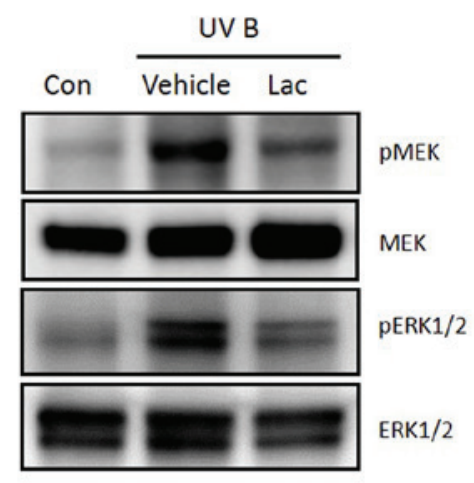

C

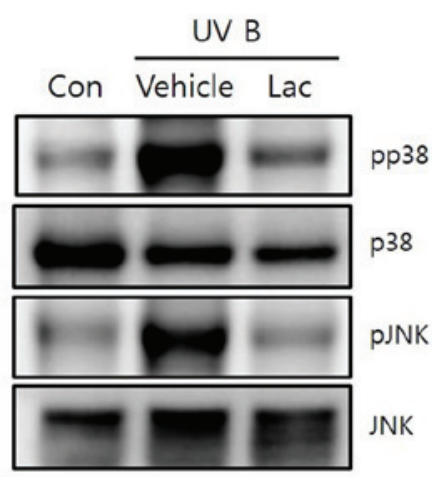

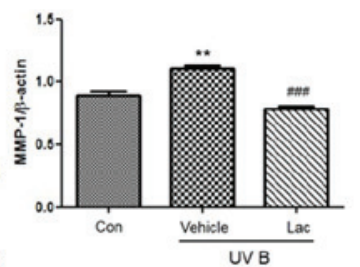
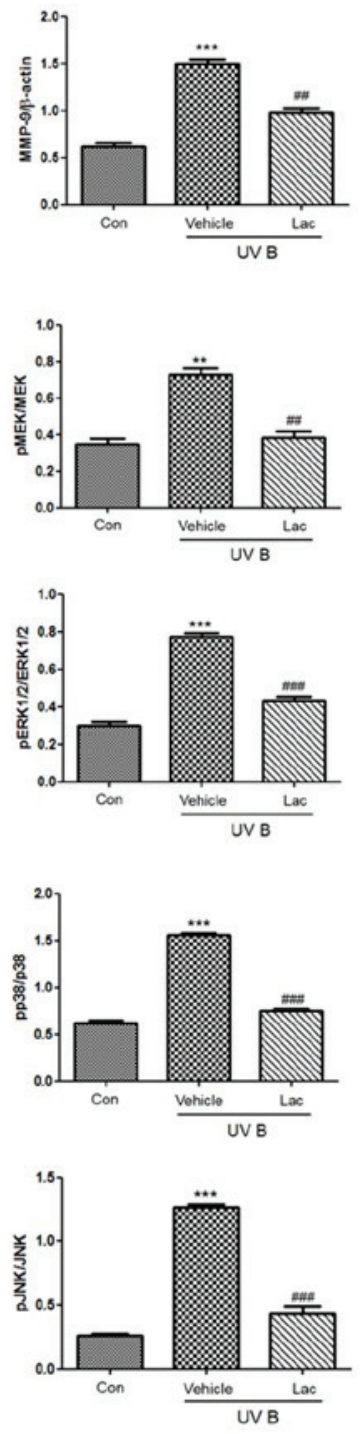

Figure 3. (A) The inhibitory effect of tyndalized Lactobacillus acidophilu on the expression of MMP-1 and MMP-9 in UVB-irradiated skin. The expression levels of the MMPs were detected by western blot analysis (B) The inhibitory effects of tyndalized L.acidophilus on the UVB-induced expression of MAPKs. Tyndalized L. acidophilus inhibited the phosphorylation of JNK 1/2, and p38. (C) Tyndalized L. acidophilus inhibited the phosphorylation of ERK or MEK, and ERK 1/2. Lac, tyndalized L. acidophilus; con, control; UV, ultraviolet; MMP, matrix metalloproteinase; JNK, Jun-amino-terminal kinase; MEK, MAPK/ERK kinase; ERK1/2, extracellular signal-regulated protein kinase $1 / 2 .{ }^{* *} \mathrm{P}<0.01,{ }^{* * * *} \mathrm{P}<0.001$ vs. the vehicle group. ${ }^{\# \#} \mathrm{P}<0.01,{ }^{\# \# \#} \mathrm{P}<0.001$ vs. the control group.

Fig. 1A). The hydration of the skin from the UVB-treated tyndalized $L$. acidophilus group mice was not reduced to the same extent as that observed in the UVB-treated vehicle group and it was significantly different, as compared with the UVB-treated vehicle group ( $\mathrm{P}<0.001$; Fig. 1A).

TEWL was significantly higher in the UVB-treated vehicle group (Fig. 1B; $\mathrm{P}<0.0001$ ), as compared with the control group. However, TEWL was significantly reduced in the UVB-treated tyndalized L.acidophilus group, as compared with the UVB-treated vehicle group $(\mathrm{P}<0.001$; Fig. 1B).

Histological evaluation of the anti-wrinkle effect of L. acidophilus in UVB-irradiated hairless mice. To determine the wrinkle-reducing effects of tyndalized L. acidophilus, skin was removed from the hairless mice and skin tissue sections were stained. H\&E staining of the skin confirmed that the thickness of the stratum corneum and the epidermis had increased in the UVB-treated vehicle group compared with the skin from the control group (Fig. 2A and B; P<0.001). Masson's trichrome staining revealed that the collagen was uniformly distributed in the dermal layer (Fig. 2C). Collagen fibers were increased in the UVB-treated tyndalized L. acidophilus group compared with the UVB-treated vehicle group (Fig. 2C). These results suggest that tyndalized L. acidophilus reduced the level of skin-wrinkling resulting from UV irradiation.

Evaluation of the anti-wrinkle effect of tyndalized L.acidophilus based on changes in epidermal thickness. To evaluate the anti-wrinkle effect of tyndalized L. acidophilus, changes in the thickness of the epidermis were investigated by measuring the distance from the keratin layer to the epidermal basement membrane in skin sections stained with H\&E using a microscope. The skin epidermal thickness was significantly increased in the UVB-treated vehicle group, as compared with the control group ( $\mathrm{P}<0.001$; Fig. 2B). The skin epidermal thickness of the the UVB-treated tyndalized L.acidophilus group was decreased by $54.1 \%$, as compared with the UVB-treated vehicle group, and this difference was statistically significant $(\mathrm{P}<0.01)$. These results suggest that tyndalized L. acidophilus is able to reduce the thickness of the epidermis and may be an effective anti-wrinkle agent.

Western blotting. UVB irradiation increased the expression of the MMPs, MMP-1 and MMP-9. Orally administered $100 \mathrm{mg}$ tyndalized L. acidophilus reduced the expression levels of MMP-1 and MMP-9 (Fig. 3A). Furthermore, tyndalized L. acidophilus treatment suppressed the UVB-induced upregulation of MMP-1 and MMP-9. The effect of tyndalized L. acidophilus on UVB-induced MAPK phosphorylation was examined in mouse skin, as MMP-9 is primarily regulated by MAPK activation. As revealed in Fig. 3B, UVB irradiation induced the phosphorylation of p38, ERK 1/2, and JNK. Pretreatment with tyndalized L. acidophilus attenuated the phosphorylation of p38, ERK1/2, JNK, and MAPK in UVB-irradiated hairless mouse skin. In addition, tyndalized L. acidophilus inhibited the UVB-induced phosphorylation of MAPK/ERK kinase (MEK; Fig. 3C). The aforementioned data suggest that tyndalized L. acidophilus protects mouse skin from UVB-induced damage.

\section{Discussion}

Photoaging refers to premature skin aging caused by chronic exposure to UV radiation, particularly the UVB component, which is regarded as the primary cause of skin damage (11). Acute or chronic exposure to UVB radiation is a major cause of dermatologic disorders. UVB irradiation of the skin induces 
acute inflammation, which is characterized by erythema, edema and immunosuppression (12). The changes that result from UV irradiation that are perceived to be associated with aging include a loss of elasticity, pigmentary changes and deep wrinkling (13). The majority of these changes arise as a result of damage to the dermis, which is visible histologically as elastotic material.

An increase in TEWL impairs the functions of the enzymes within the skin and this results in visibly dry and aged skin (14). UVB irradiation causes skin damage, which leads to skin dehydration and increases in TEWL. High TEWL values, which are indicative of disturbances in the skin's barrier function, are frequently correlated with low levels of hydration of the stratum corneum (15). In the current study, tyndalized L. acidophilus effectively reduced skin damage, and this was associated with increases in skin hydration and decreases in TEWL.

Wrinkle formation is associated with the regulation of collagen synthesis and degradation. As marked degenerative changes are observed in the extracellular matrix (ECM) of the dermis during wrinkle formation, ECM-degrading enzymes are considered to be involved in wrinkle formation (16). The effects of tyndalized L. acidophilus on wrinkle formation caused by UVB irradiation were investigated in replicas of the dorsal skin of the mice.

Photoaging is characterized by the degradation of collagen and the accumulation of abnormal elastin in the superficial dermis (17). Epidermal thickness is used to quantify skin photoaging as epidermal hypertrophy has been hypothesized to cause wrinkle formation (18). In the present study, H\&E staining and Masson's trichrome staining demonstrated the effects of tyndalized L. acidophilus on changes within the dorsal skin histologically. H\&E staining revealed that UVB irradiation increased the thickness of the epidermis, and that the administration of tyndalized L. acidophilus alleviated this effect.

MMPs form a family of enzymes that possess ECM-degrading properties. UVB irradiation is known to MMP synthesis (19). UVB irradiation induces MMP production by activating cellular signaling transduction pathways (19). Each member of the MMP family has a unique function in response to the skin undergoing irradiation by UVB, and MMP-1 serves an important role in the degradation of the ECM that is caused by photoaging (20). Previous research has demonstrated that decursin inhibits the UVB-induced expression levels of MMP in human dermal fibroblasts by regulating nuclear factor- $\kappa \mathrm{B}(21)$. In the current study, it was revealed that UVB irradiation led to increases in the expression levels of MMP-1 and MMP-9, and that the administration of tyndalized L. acidophilus reduced the expression of MMP-1 and MMP-9 proteins.

p38 MAPK is a member of a highly conserved family of serine/threonine protein kinases that includes ERK and c-JNK. Various stressors, such as UV irradiation, oxidative injury, heat shock, cytokines and other pro-inflammatory stimuli, lead to the induction of the p38 MAPK-dependent signaling cascade (22). Major signaling pathways known to mediate UVB-induced biological responses involve MAPKs (23). The present study investigated whether the inhibitory effect of tyndalized L. acidophilus was associated with the downregulation of the MAPK family of protein kinases and MEK. It was determined that the expression levels of JNK, p38, and ERK increased in UVB-irradiated skin, and that tyndalized L. acidophilus attenuated the elevations in the expression levels of the aforementioned proteins.

In conclusion, tyndalized L. acidophilus effectively inhibited wrinkle formation induced by UVB irradiation, and this inhibition was attributed to the downregulation of MMPs. Therefore, tyndalized L. acidophilus may serve as a potential agent for preventing skin photoaging and wrinkle formation.

\section{Acknowledgements}

The present study was supported by a grant from the Korea Institute of Oriental Medicine (grant no. K14101).

\section{References}

1. Uitto $\mathrm{J}$ and Bernstein EF: Molecular mechanisms of cutaneous aging: Connective tissue alterations in the dermis. J Investig Dermatol Symp Proc 3: 41-44, 1998.

2. Pontius AT and Smith PW: An antiaging and regenerative medicine approach to optimal skin health. Facial Plast Surg 27: 29-34, 2011.

3. Yano K, Kajiya K, Ishiwata M, Hong YK, Miyakawa T and Detmar M: Ultraviolet B-induced skin angiogenesis is associated with a switch in the balance of vascular endothelial growth factor and thrombospondin-1 expression. J Invest Dermatol 122: 201-208, 2004.

4. Moriwaki S and Takahashi Y: Photoaging and DNA repair. J Dermatol Sci 50: 169-176, 2008.

5. Masaki H, Atsumi T and Sakurai H: Protective activity of hamamelitannin on cell damage of murine skin fibroblasts induced by UVB irradiation. J Dermatol Sci 10: 25-34, 1995.

6. Rabe JH, Mamelak AJ, McElgunn PJ, Morison WL and Sauder DN: Photoaging: Mechanisms and repair. J Am Acad Dermatol 55: 1-19, 2006.

7. Tanriover MD, Aksoy DY and Unal S: Use of probiotics in various diseases: Evidence and promises. Pol Arch Med Wewn 122 (Suppl 1): S72-S77, 2012.

8. Meneghin F, Fabiano V, Mameli C and Zuccotti GV: Probiotics and atopic dermatitis in children. Pharmaceuticals (Basel) 5: 727-744, 2012.

9. Inoue $\mathrm{Y}$, Kambara $\mathrm{T}$, Murata N, Komori-Yamaguchi J, Matsukura S, Takahashi Y, Ikezawa Z and Aihara M: Effects of oral administration of Lactobacillus acidophilus L-92 on the symptoms and serum cytokines of atopic dermatitis in Japanese adults: A double-blind, randomized, clinical trial. Int Arch Allergy Immunol 165: 247-254, 2014.

10. Sunada Y, Nakamura S and Kamei C: Effect of Lactobacillus acidophilus strain L-55 on the development of atopic dermatitis-like skin lesions in $\mathrm{NC} / \mathrm{Nga}$ mice. Int Immunopharmacol 8: 1761-1766, 2008.

11. Pyun HB, Kim M, Park J, Sakai Y, Numata N, Shin JY, Shin HJ, Kim DU and Hwang JK: Effects of collagen tripeptide supplement on photoaging and epidermal skin barrier in UVB-exposed hairless mice. Prev Nutr Food Sci 17: 245-253, 2012.

12. Lee $\mathrm{CH}, \mathrm{Wu} \mathrm{SB}$, Hong $\mathrm{CH}$, Yu HS and Wei YH: Molecular mechanisms of UV-induced apoptosis and its effects on skin residential cells: The implication in UV-based phototherapy. Int J Mol Sci 14: 6414-6435, 2013.

13. Inomata S, Matsunaga Y, Amano S, Takada K, Kobayashi K, Tsunenaga M, Nishiyama T, Kohno Y and Fukuda M: Possible involvement of gelatinases in basement membrane damage and wrinkle formation in chronically ultraviolet B-exposed hairless mouse. J Invest Dermatol 120: 128-134, 2003.

14. Verdier-Sévrain S and Bonté F: Skin hydration: A review on its molecular mechanisms. J Cosmet Dermatol 6: 75-82, 2007.

15. Proksch E, Brandner JM and Jensen JM: The skin: An indispensable barrier. Exp Dermatol 17: 1063-1072, 2008.

16. Kligman LH, Gebre M, Alper R and Kefalides NA: Collagen metabolism in ultraviolet irradiated hairless mouse skin and its correlation to histochemical observations. J Invest Dermatol 93: 210-214, 1989. 
17. Vayalil PK, Mittal A, Hara Y, Elmets CA and Katiyar SK: Green tea polyphenols prevent ultraviolet light-induced oxidative damage and matrix metalloproteinases expression in mouse skin. J Invest Dermatol 122: 1480-1487, 2004.

18. Urikura I, Sugawara T and Hirata T: Protective effect of fucoxanthin against UVB-induced skin photoaging in hairless mice. Biosci Biotechnol Biochem 75: 757-760, 2011.

19. Kim SR, Jung YR, An HJ, Kim DH, Jang EJ, Choi YJ, Moon KM, Park $\mathrm{MH}$, Park $\mathrm{CH}$, Chung $\mathrm{KW}$, et al: Anti-wrinkle and anti-inflammatory effects of active garlic components and the inhibition of MMPs via NF-кB signaling. PLoS One 8: e73877, 2013.

20. Ho JN, Lee YH, Park JS, Jun WJ, Kim HK, Hong BS, Shin DH and Cho HY: Protective effects of aucubin isolated from eucommia ulmoides against UVB-induced oxidative stress in human skin fibroblasts. Biol Pharm Bull 28: 1244-1248, 2005.
21. Hwang BM, Noh EM, Kim JS, Kim JM, Hwang JK, Kim HK, Kang JS, Kim DS, Chae HJ, You YO, et al: Decursin inhibits UVB-induced MMP expression in human dermal fibroblasts via regulation of nuclear factor- $\kappa \mathrm{B}$. Int J Mol Med 31: 477-483, 2013.

22. Kim AL, Labasi JM, Zhu Y, Tang X, McClure K, Gabel CA, Athar M and Bickers DR: Role of p38 MAPK in UVB-induced inflammatory responses in the skin of SKH-1 hairless mice. J Invest Dermatol 124: 1318-1325, 2005.

23. Bode AM and Dong Z: Mitogen-activated protein kinase activation in UV-induced signal transduction. Sci STKE 2003: RE2, 2003. 